\title{
Recent advances in gelatin-based therapeutics
}

Echave, Mari Carmen; Hernáez-Moya, Raquel; Iturriaga, Leire; Pedraz, José Luis; Lakshminarayanan, Rajamani; Dolatshahi-Pirouz, Alireza; Taebnia, Nayere; Orive, Gorka

\section{Published in:}

Expert Opinion on Biological Therapy

Link to article, DOI:

$10.1080 / 14712598.2019 .1610383$

Publication date:

2019

Document Version

Peer reviewed version

Link back to DTU Orbit

Citation (APA):

Echave, M. C., Hernáez-Moya, R., Iturriaga, L., Pedraz, J. L., Lakshminarayanan, R., Dolatshahi-Pirouz, A., Taebnia, N., \& Orive, G. (2019). Recent advances in gelatin-based therapeutics. Expert Opinion on Biological Therapy, 19(8), 773-779. https://doi.org/10.1080/14712598.2019.1610383

\section{General rights}

Copyright and moral rights for the publications made accessible in the public portal are retained by the authors and/or other copyright owners and it is a condition of accessing publications that users recognise and abide by the legal requirements associated with these rights.

- Users may download and print one copy of any publication from the public portal for the purpose of private study or research.

- You may not further distribute the material or use it for any profit-making activity or commercial gain

- You may freely distribute the URL identifying the publication in the public portal 


\title{
Recent advances in gelatin-based therapeutics
}

\author{
Mari Carmen Echave, Raquel Hernáez-Moya, Leire Iturriaga, José Luis \\ Pedraz, Rajamani Lakshminarayanan, Alireza Dolatshahi-Pirouz, Nayere \\ Taebnia \& Gorka Orive
}

To cite this article: Mari Carmen Echave, Raquel Hernáez-Moya, Leire Iturriaga, José Luis Pedraz, Rajamani Lakshminarayanan, Alireza Dolatshahi-Pirouz, Nayere Taebnia \& Gorka Orive (2019): Recent advances in gelatin-based therapeutics, Expert Opinion on Biological Therapy, DOI: 10.1080/14712598.2019.1610383

To link to this article: https://doi.org/10.1080/14712598.2019.1610383
央 Accepted author version posted online: 22 Apr 2019.

Submit your article to this journal


Publisher: Taylor \& Francis

Journal: Expert Opinion on Biological Therapy

DOI: $10.1080 / 14712598.2019 .1610383$

\section{Recent advances in gelatin-based therapeutics}

Mari Carmen Echave ${ }^{a, b}$, Raquel Hernáez-Moya ${ }^{a, b}$, Leire Iturriaga ${ }^{a, b}$, José Luis Pedraz $^{\mathrm{a}, \mathrm{b}}$, Rajamani Lakshminarayanan ${ }^{\mathrm{c}, \mathrm{d}}$, Alireza Dolatshahi-Pirouz ${ }^{\mathrm{e}}$, Nayere Taebnia $^{\mathrm{e}}$, Gorka Orive ${ }^{\mathrm{a}, \mathrm{b}, \mathrm{f}, \mathrm{g}^{*}}$

Affiliations:

a NanoBioCel Group, Laboratory of Pharmaceutics, School of Pharmacy, University of the Basque Country UPV/EHU, Paseo de la Universidad 7, Vitoria-Gasteiz 01006, Spain.

${ }^{\mathrm{b}}$ Biomedical Research Networking Centre in Bioengineering, Biomaterials and Nanomedicine (CIBER-BBN). Vitoria-Gasteiz, Spain.

${ }^{\mathrm{C} A n t i-I n f e c t i v e s ~ R e s e a r c h ~ G r o u p, ~ S i n g a p o r e ~ E y e ~ R e s e a r c h ~ I n s t i t u t e, ~ T h e ~ A c a d e m i a, ~} 20$ College Road, Discovery Tower, Singapore.

'Ophthalmology and Visual Sciences Academic Clinical Program, Duke-NUS Graduate Medical School, Singapore

${ }^{\mathrm{e}}$ Technical University of Denmark, DTU Nanotech, Center for Intestinal Absorption and Transport of Biopharmaceutical, 2800 Kgs, Denmark.

fUniversity Institute for Regenerative Medicine and Oral Implantology - UIRMI (UPV/EHU-Fundación Eduardo Anitua), Vitoria, Spain; BTI Biotechnology Institute, Vitoria, Spain.

'Singapore Eye Research Institute, The Academia, 20 College Road, Discovery Tower, Singapore.

${ }^{*}$ Corresponding author:

Gorka Orive (gorka.orive@ehu.eus). Phone: +34 663027696

Twitter: @gorka_orive 


\begin{abstract}
Introduction: Biomaterials have provided a wide range of exciting opportunities in tissue engineering and regenerative medicine. Gelatin, a collagen-derived natural biopolymer, has been extensively used in regenerative medicine applications over the years, due to its cell-responsive properties and the capacity to deliver a wide range of biomolecules.
\end{abstract}

Areas covered: The most relevant properties of gelatin as biomaterial are presented together with its main therapeutic applications. The latter includes drug delivery systems, tissue engineering approaches, potential uses as ink for 3D/4D Bioprinting and its relevance in organ-on-a-chip platforms.

Expert Opinion: Advances in polymer chemistry, mechanobiology, imaging technologies and 3D biofabrication techniques have expanded the application of gelatin in multiple biomedical research applications ranging from bone and cartilage tissue engineering, to wound healing and anti-cancer therapy. Here, we highlight the latest advances in gelatin-based approaches within the fields of biomaterial-based drug delivery and tissue engineering together with some of the most relevant challenges and limitations. 
Keywords: Biomaterial, Drug Delivery, Gelatin, Regenerative Medicine, Tissue Engineering

\section{Article highlights}

- Gelatin is a collagen-derived protein with cell-responsive properties.

- Gelatin creates polyion complexes with charged therapeutic compounds.

- Crosslinked gelatin scaffolds are used for many 3D cell cultures in regenerative medicine.

- Gelatin is used in the fabrication of micro/nanocapsules for drug delivery. 


\section{Introduction}

Biomaterials include a broad class of substances that interface with biological entities and have over the decades provided a wide range of breakthrough opportunities in regenerative medicine. Our steadily increasing knowledge in biology, pharmacy, chemistry and material science, together with the interaction of these disciplines with one another, is rapidly expanding the application of biomaterials in the areas of controlled drug delivery, prosthetics and tissue engineering [1]. Among the many different types of biomaterials, bioactive polymers have advantages over their classic counterparts (i.e., static and nonstimulatory) as they are capable of playing an active role in drug delivery, control cell fate, regulate cell organoid formation and function for various drug testing applications, and even drive tissue repair and regeneration within the body $[2,3]$.

The field of biomaterials has even more progressed due to a variety of prominent advancements in chemistry, cutting-edge imaging technologies, novel cell sources, avant-garde and optimized drug delivery platforms, increased knowledge on mechanobiology [4] (the process by which physical forces are converted into biochemical signals) and advances in various biofabrication processes such as three-dimensional (3D) bioprinting [5] and selfassembly technologies.

Gelatin is one of these particularly fruitful biomaterials, holding enormous promise for the above mentioned biomedical applications. This natural polymer derived from collagen, contains enjoys integrin binding cell adhesion peptides [6] and metalloproteinase (MMP)-sensitive peptide sequences allowing celltriggered degradation. Besides, one of the main features of this water-soluble 
protein is its thermo-responsive character, undergoing a reversible sol-gel transition when cooled upper its critical solution temperature $\left(25-35^{\circ} \mathrm{C}\right)$. Since gelatin provides a biologically active 3D microenvironment for regulating cell viability, growth and differentiation, it has attracted a great deal of interest nowadays either as scaffolds for tissue regeneration or carriers for controlled drug delivery. Currently there are several gelatin-based medical devices marketed by companies worldwide. For example, Gelita-SPON®, Cutanplast $₫$, Gelfoam $®$ and SurgiFoam $®$ are absorbable gelatin sponges indicated to be used as hemostats in surgical procedures such as neurosurgeries, thoracic surgeries or ocular surgeries. More interestingly, gelatin containing allograft product has been approved by Food and Drug Administration for bone tissue engineering purposes. DBX® Strips is a flexible and bendable osteoinductive tape composed of demineralized bone matrix, gelatin and sodium hyaluronate, and it is indicated as a bone void filler. Moreover, a novel gelatin surgical implant, $X E N \otimes$ Gel Stent, has been developed to safely and effectively reduce the high eye pressure in refractory glaucoma cases. Regarding the commercialization of gelatin containing medicines, strict safety and quality requirements are demanded from the manufacturers involved in the production of gelatin for human use. The main objective of this rigorous control is to completely avoid the risk of bovine spongiform encephalopathy associated with the use of animal origin raw materials. The pharmaceutical grade gelatin must comply not only with the current requirements for edible gelatin, but also with the provisions for medical products. In this regard, the European Pharmacopoeia lists specific requisite necessary for the manufacture of pharmaceutical grade gelatin. 
In this review, we highlight the potential applications of gelatin in biomedicine and describe the corresponding advances in drug delivery and tissue engineering.

\section{Gelatin-based biomaterials}

Due to its cell-stimulatory properties, gelatin has been widely used in regenerative medicine and tissue engineering. Gelatin is a natural origin polymer, which holds several advantages over its precursor collagen. The low water-solubility of collagen under neutral conditions is one of the main limitations for biomedical purposes [7]. This drawback of collagen can be

overcome with the extraction process of gelatin. Another notable property of gelatin is its ability to create poly-ionic complexes with charged therapeutic compounds such as proteins, growth factors, nucleotides and polysaccharides [8], which in turn makes it ideal as a delivery vehicle for a broad range of biomolecules (Figure 1). In fact, gelatin exhibits depending on its extraction conditions either a net positive (IEP=9, Type A gelatin) or net negative $($ IEP $=5$, Type B gelatin) isoelectric point at $\mathrm{pH} 7.4$, which allows sequestering oppositely charged proteins while maintaining its bioactivity. As a consequence, gelatin is a primary material in the fabrication of microcapsules and microspheres for drug delivery [9]. Furthermore, the source and the extraction conditions are key factors for obtaining gelatins with diverse physicochemical properties (melting temperature, gel modulus or viscosity), due to the differences in the amino acids proportions and the molecular weights of the resulting materials [10]. On the other hand, gelatin is easily functionalized to fabricate materials with tailor-made features, opening new therapeutic applications. 
As an example, gelatin-based 3D microgels can be used to stimulate cell proliferation and differentiation of various encapsulated cells, such as stem cells, and can also improve the regenerative impact of injected cell-laden microbeads in lesion sites [11]. Over and above, these microgels can shield the cells from shear-force associated mortality during injection, and provide them with a milieu that enhances cell retention within the targeted site.

Crosslinked gelatin scaffolds can be used for 3D cell culturing. However, thermal gelation of gelatin typically results in frail and weak gels. To address this, conventional chemical procedures are applied to develop covalently crosslinked gelatin-based hydrogels [12]. One of the striking strategies proposed by several researchers has been the enzymatic crosslinking of gelatin systems, using various enzymes such as horseradish peroxidase [13] or microbial transglutaminase [14]. These systems have been evaluated for different tissue engineering purposes, since versatile hydrogels with tunable gelation rate and final mechanical strength have been successfully developed. As an example, injectable enzymatically crosslinked gelatinhydroxyphenylpropionic acid composite hydrogel have been evaluated both for cartilage [15] and brain [16] tissue reparation. Furthermore, photocrosslinkable gelatin methacrylate (GeIMA) hydrogels have garnered great interest in various therapeutic applications, ranging from corneal tissue engineering [17], to peripheral nerve regeneration [18] and cartilage construct fabrication [19]. Furthermore, injectable covalently crosslinked gelatin hydrogels have been recently developed with the aid of pendant tetrazine or norbornene click chemistry pairs in modified polymers [20]. These gelatin polymers rapidly crosslink in combination and they start to degrade when injected in vivo. 
Moreover, they promote high cell viability and have the capacity to drive encapsulated cells into 3D elongated morphologies.

The ongoing progression in 3D bioprinting enables unprecedented control over spatial distribution of materials, cells and biomolecules and ultimately facilitate the fabrication of more native-like 3D tissue structures. In one noteworthy example, Jia $\mathrm{W}$ and colleagues designed a direct 3D multilayered coaxial extrusion printing strategy to develop highly perfusable and organized vessels. The blended bioink consisted of sodium alginate, gelatin methacrylate and 4-arm poly (ethylene glycol)-tetra-acrylate (PEGTA). This double crosslinked (covalently and ionically) system allowed the proliferation and propagation of encapsulated endothelial and mesenchymal stem cells inside the 3D printed scaffolds and ultimately facilitated the formation of native-like perfusable vessels [21].

\section{Progress in drug delivery}

Gelatin-based biomaterials are of great interest in the design and fabrication of drug delivery systems, which provide controlled, sustained and/or targeted release of bioactive molecules, while enhancing their bioavailability and improving their therapeutic effects. This modern strategy is currently employed for several biomedical applications, ranging from tissue regeneration and wound healing to anti-cancer treatments and medical imaging. Wound healing is a highly complex dynamic process during which a damaged or injured tissue is repaired or replaced. Skin wounds are mostly due to trauma, surgery or burns; however, diseases such as diabetes may give rise to a prolonged healing time [22]. Gelatin-based dressings provide high protection capacity against infections and can accelerate the wound healing process, thanks to their tailorable 
mechanical and degradation properties. More than that, gelatin also enjoys favorable biocompatibility and contributes to a balanced hydrophobicity/hydrophilicity of the wound-dressing, resulting in a suitable release of biomolecules. As an interesting example, gelatin was combined with

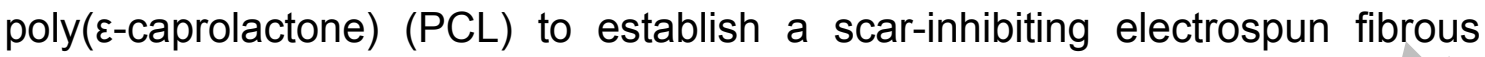
scaffold, loaded with transforming growth factor $\beta 1$ (TGF- $\beta 1$ ) inhibitor (Figure 2) [23]. Through this approach, fibroblast over-proliferation was effectively inhibited in vitro, and scarring was successfully prevented in vivo during a wound-healing process in rabbit ear model. In another recent study, a conductive composite scaffold was fabricated based on PCL/gelatin nanofibers and silicate-based bioceramic particles [24]. In this system the nanofibrous microstructure of the scaffolds mimicked that of the extracellular matrix, and in combination with the sustained release of silicon ions from the silicate-based ceramic, a synergistic and beneficial effect in diabetic wound healing was obtained.

The release kinetics of bioactive molecules and drugs is highly dependent on degradation rate and water uptake of the gelatin networks. These properties represent a great opportunity for preventing multidrug-resistant bacterial infections, by releasing antibiotics in a controlled manner $[25,26]$. For instance, Lee and colleagues introduced gelatin-hydroxypropionic acid hydrogels, formed in the presence of bactericidal $\mathrm{H}_{2} \mathrm{O}_{2}$ as antimicrobial injectable or sprayable dressings [26]. Other potential application of gelatin-based drug delivery systems is in cancer therapy, where improving drug bioavailability and targeted delivery have always been a major practical concern. For instance, the combined administration of dendritic cells (DCs) and oncolytic adenovirus in a tumor environment has a potent antineoplastic immune effect. However, the 
rapid inactivation of the drugs within the tumor site could reduce their effectiveness. To address this challenge, injectable and biodegradable scaffolds based on gelatin have been developed, enabling an extended delivery of both DCs and oncolytic adenovirus [27].

Last but not least, gelatin-based micro and nanoparticles are emerging as promising flexible and powerful biomaterials for growth factor delivery. These characteristics are opening up new paths toward therapeutic alternatives in various medical fields such as cancer treatment, neuroprotection after postischemic brain injury [28] and immunization. As an example, Sabet S. and colleagues reported gelatin nanoparticles as a non-viral vaccine and gene delivery system for hepatitis $\mathrm{C}$, which effectively transferred the nonstructural protein 2 gene into bacterial cells [29].

\section{New advances in tissue engineering}

The overarching goal in tissue engineering is to develop artificial systems or structures that can recapitulate some of the most important functions of native tissues. However, this goal is challenging due to the extremely complex tissue architectures in the body, together with the many synergistic biological components within native tissues. The latest efforts in the field are currently directed towards combining different types of biomaterials to generate nativelike synergistic effects, as well as providing the much-needed native-like tissue microarchitectures. Through this approach, several potential systems based on

gelatin have been developed over the past decade. For instance, the combination of gelatin with hyaluronic acid was introduced as a biocomponentbased hydrogel to provide elasticity and promote vascularization of various target tissues [30]. 
Non-union bone fractures cause long-term incapacity and pain. Indeed, only in the United States around 8 million people suffers from bone injuries each year and conventional treatments are not effective in at least $10 \%$ of the cases. Several studies have recently emerged on new alternatives and bioactive agents for bone tissue engineering. In an intriguing approach, platelet loaded chitosan-gelatin composite hydrogel was applied to increased bone regeneration in bilateral critical-sized radial bone defect model in rats [31]. Furthermore, enhanced mRNA levels of alkaline phosphatase, as well as osteogenic (collagen type 1, osteocalcin, CD31 and run related transcription factor 2) and angiogenic (vascular endothelial growth factor) differentiation markers were also observed.

Even though gelatin-based scaffolds have not yet been commercialized for bone regeneration applications, the combination of gelatin with calcium phosphate ceramics and other synthetic polymers stand as promising options for clinical applications [32]. One of the biggest challenges here is to mold biomaterials into the similar complex architectures as those seen in bone defects. To overcome this limitation, several strategies have been studied including injectable systems [33] and 3D printed implantable structures [34], that facilitate fabrication of customized engineered scaffolds, that can perfectly adapt into bone defects (Figure 3).

In addition, new avenues are provided by tissue engineering approaches to facilitate the repair of the native cartilage tissue, which naturally presents limited capacity for spontaneous repair. Over the last few years, several biomaterials including gelatin have been investigated and tested for cartilage tissue engineering applications. For example, the chondrogenesis of stem cells has 
been induced by co-culturing bone marrow stromal cell and chondrocytes in electrospun gelatin/PCL nanofibrous biomaterials [35]. In another approach, multipotent articular cartilage-resident chondroprogenitor cells were embedded in gelatin hydrogels to create cartilage-tissue regeneration [36]. These encapsulated cells presented a better profile in terms of neo cartilage production compared to differentiated chondrocytes. In addition, higher expression levels of the lubricating factor, PRG4, and lower expression levels of collagen type $\mathrm{X}$ hypertrophy marker confirmed the regenerative potential of these cells.

\section{Looking into the future}

Gelatin holds great promise for regenerative medicine, thanks to its biocompatibility and novel biomedical approaches such as cell-laden gelatinbased 3D tissue models are expected to be developed in near future to assist better understanding of diseases or drug development and screening. These 3D microphysiological structures could potentially complement or even replace current in vivo studies. As an example, gelatin microparticles have been combined with pancreatic cancer cells and fibroblasts to replicate the complexity of the pancreatic tumor microenvironment and to clarify the complex stroma-cancer inter-relationship [37]. In another intriguing approach, human cardiac microtissues have been bioengineered using cardiomyocytes derived from human induced pluripotent stem cells, encapsulated in gelatin hydrogels with tunable stiffness and degradation rates [38]. As a proof-of-concept Agrawal and colleagues designed a 3D skeletal muscle-on-a-chip platform with cellladen gelatin hydrogel as a screening platform for drugs and toxics such as 
cardiotoxin [39], which can also be applied for preclinical drug discovery and development.

\section{Conclusion}

Gelatin offers a broad spectrum of applications and possibilities ranging from micro/nanoparticle-based drug delivery to tissue repair and regeneration by means of 3D biomimetic scaffolds. We envision that the ongoing progression of research in this area will promote the future translation of gelatin-based biomaterials into the clinics.

\section{Expert Opinion}

Gelatin is well-known biomaterial with exciting properties for protein and drug delivery and tissue repair and regeneration. Furthermore, it can be easily tailored to achieve different grades of mechanical stability and stiffness, being also an excellent candidate for controlling cell behavior, cell differentiation and even for cell transplantation. Gelatin can be used alone or combined with other biomaterials as bioink for 3D/4D bioprinting, opening new fields in the design and development of novel spatially customized anatomical structures in a personalized manner.

The use of gelatin however may see new horizons in the next few years. We envision that its properties may help to develop 2D and 3D nerve constructs based on the mechanical properties and conductivity of novel gelatin-based composites. This progress may move forward its use in other cell-platforms such as organ-on-a-chip devices, that is, multi-channel 3D microfluidic cell culture chips by which it is possible to understand tissue and organ functions as well and test and screen a wide range of drugs. In addition, gelatin-based 
composites together with differentiated human stem cells may bring new hopes to heal spinal cord injuries and to limit the secondary damage.

Another interesting therapeutic window for gelatin relies on its potential use in transdermal delivery of therapeutics. By using biocompatible, biodegradable and bioresponsive gelatin-based microneedles, scientists are churning out new platforms for drug delivery across the skin in a safe and cost-efficient way. Last but not least, gelatin has been successfully combined and mixed with autologous growth factors derived from human plasma and platelets. Due its ability to sequester oppositely charged proteins while maintaining its bioactivity, gelatin can provide additional properties to the naturally forming fibrin scaffold and thus be an aid in the therapeutic use of platelet rich plasma, an approach that is being used in multiple medical fields including dentistry, orthopedics, dermatology and ophthalmology.

Nevertheless, the use and applicability of this interesting material presents several challenges. The weak mechanical properties of gelatin may limit certain applications, especially those in which higher mechanical responses are demanding. To address this, both composite systems and chemical modifications are being explored. For example, aminoacidic structure of gelatin has been modified with the incorporation of diverse chemical motifs such as methacryloyl, catechol, phenol or epigallactocachecin gallate groups to increase its mechanical properties and durability.

In summary, gelatin provides a wide range of uses and applications in many different therapeutic fields. Progress in polymer chemistry, cell biology, mechanobiology, imaging technologies and 3D biofabrication techniques are 
expanding its value and potential in drug delivery and tissue repair and regeneration approaches.

\section{Funding}

This paper was not funded.

\section{Declaration of Interests}

G Orive would like to acknowledge funding from project SAF2016-76150-R from the Spanish Ministry of Economy, Industry and Competitiveness and intellectual and technical assistance from the ICTS "NANBIOSIS", more specifically by the Drug Formulation Unit (U10) of the CIBER in Bioengineering, Biomaterials \& Nanomedicine (CIBER-BBN) at the University of the Basque Country (UPV/EHU). G Orive also acknowledgesfinancial support from the Basque Country Government (Grupos Consolidados, No ref: IT907-16, ELKARTEK 16/77). MC Echave MC gives thanks to the Basque Country Government (Departamento de Educación, Universidades e Investigación) for a granted fellowship. A Dolatshahi-Pirouz .acknowledges grant support from the Danish Council for Independent Research (Technology and Production Sciences, 5054-00142B), Danish Council for Independent Research (Technology and Production Sciences, 8105-00003B), Gigtforeningen (R139A3864), and the Villum Foundation (10103). The authors have no other relevant affiliations or financial involvement with any organization or entity with a financial interest in or financial conflict with the subject matter or materials discussed in the manuscript apart from those disclosed. 


\section{Reviewer Disclosures}

Peer reviewers on this manuscript have no relevant financial relationships or otherwise to disclose.

\section{References}

[1] Tibbitt MW, Langer R. Living Biomaterials. Acc.Chem.Res. 2017;50(3):508513.

[2] Darnell M, Mooney DJ. Leveraging Advances in Biology to Design Biomaterials. Nat.Mater. 2017;16(12):1178-1185.

[3] Santos E, Hernandez RM, Pedraz JL, et al. Novel Advances in the Design of Three-Dimensional Bio-Scaffolds to Control Cell Fate: Translation from 2D to 3D. Trends Biotechnol. 2012;30(6):331-341.

[4] Li L, Eyckmans J, Chen CS. Designer Biomaterials for Mechanobiology. Nat.Mater. 2017;16(12):1164-1168.

[5] Kolesky DB, Homan KA, Skylar-Scott MA, et al. Three-Dimensional Bioprinting of Thick Vascularized Tissues. Proc.Natl.Acad.Sci.U.S.A. 2016;113(12):3179-3184.

[6] Young S, Wong M, Tabata Y, et al. Gelatin as a Delivery Vehicle for the Controlled Release of Bioactive Molecules. J.Control.Release. 2005;109(13):256-274.

[7] Zhang M, Ding C, Yang J, et al. Study of Interaction between Water-Soluble Collagen and Carboxymethyl Cellulose in Neutral Aqueous Solution. Carbohydr.Polym. 2016;137:410-417.

[8] Larsen M, Artym VV, Green JA, et al. The Matrix Reorganized: Extracellular Matrix Remodeling and Integrin Signaling. Curr.Opin.Cell Biol. 2006;18(5):463471.

[9] Diba M, Pape B, Klymov A, et al. Nanostructured Raspberry-Like Gelatin Microspheres for Local Delivery of Multiple Biomolecules. Acta Biomater. 2017;58:67-79.

[10] Karim AA, Bhat R. Fish Gelatin: Properties, Challenges, and Prospects as an Alternative to Mammalian Gelatins. Food Hydrocolloids. 2009;23(3):563-576.

[11] Li F, Truong VX, Thissen H, et al. Microfluidic Encapsulation of Human Mesenchymal Stem Cells for Articular Cartilage Tissue Regeneration. ACS Appl.Mater.Interfaces. 2017;9(10):8589-8601. 
[12] Nichol JW, Koshy ST, Bae H, et al. Cell-Laden Microengineered Gelatin Methacrylate Hydrogels. Biomaterials. 2010;31(21):5536-5544.

[13] Lim TC, Rokkappanavar S, Toh WS, et al. Chemotactic Recruitment of Adult Neural Progenitor Cells into Multifunctional Hydrogels Providing Sustained SDF-1alpha Release and Compatible Structural Support. FASEB J. 2013;27(3):1023-1033.

[14] Zhou M, Lee BH, Tan YJ, et al. Microbial Transglutaminase Induced Controlled Crosslinking of Gelatin Methacryloyl to Tailor Rheological Properties for 3D Printing. Biofabrication. 2019;

[15] Wang LS, Du C, Toh WS, et al. Modulation of Chondrocyte Functions and Stiffness-Dependent Cartilage Repair using an Injectable Enzymatically Crosslinked Hydrogel with Tunable Mechanical Properties. Biomaterials. 2014;35(7):2207-2217.

[16] Lim TC, Toh WS, Wang LS, et al. The Effect of Injectable GelatinHydroxyphenylpropionic Acid Hydrogel Matrices on the Proliferation, Migration, Differentiation and Oxidative Stress Resistance of Adult Neural Stem Cells. Biomaterials. 2012;33(12):3446-3455.

[17] Rizwan M, Peh GS, Ang HP, et al. Sequentially-Crosslinked Bioactive Hydrogels as Nano-Patterned Substrates with Customizable Stiffness and Degradation for Corneal Tissue Engineering Applications. Biomaterials. 2017;120:139-154.

[18] Uz M, Buyukoz M, Sharma AD, et al. Gelatin-Based 3D Conduits for Transdifferentiation of Mesenchymal Stem Cells into Schwann Cell-Like Phenotypes. Acta Biomater. 2017;53:293-306.

[19] Boere KW, Visser J, Seyednejad H, et al. Covalent Attachment of a ThreeDimensionally Printed Thermoplast to a Gelatin Hydrogel for Mechanically Enhanced Cartilage Constructs. Acta Biomater. 2014;10(6):2602-2611.

[20] Koshy ST, Desai RM, Joly P, et al. Click-Crosslinked Injectable Gelatin Hydrogels. Adv. Healthc. Mater. 2016;5(5):541-547.

[21] Jia W, Gungor-Ozkerim PS, Zhang YS, et al. Direct 3D Bioprinting of Perfusable Vascular Constructs using a Blend Bioink. Biomaterials. 2016;106:58-68.

[22] Zhao X, Sun X, Yildirimer L, et al. Cell Infiltrative Hydrogel Fibrous Scaffolds for Accelerated Wound Healing. Acta Biomater. 2017;49:66-77.

[23] Wang L, Yang J, Ran B, et al. Small Molecular TGF-Beta1-Inhibitor-Loaded Electrospun Fibrous Scaffolds for Preventing Hypertrophic Scars. ACS Appl.Mater.Interfaces. 2017;9(38):32545-32553. 
[24] Lv F, Wang J, Xu P, et al. A Conducive Bioceramic/Polymer Composite Biomaterial for Diabetic Wound Healing. Acta Biomater. 2017;60:128-143.

[25] Dhand C, Venkatesh M, Barathi VA, et al. Bio-Inspired Crosslinking and Matrix-Drug Interactions for Advanced Wound Dressings with Long-Term Antimicrobial Activity. Biomaterials. 2017;138:153-168.

[26] Lee Y, Choi KH, Park KM, et al. In Situ Forming and H2O2-Releasing Hydrogels for Treatment of Drug-Resistant Bacterial Infections. ACS Appl.Mater.Interfaces. 2017;9(20):16890-16899.

[27] Oh E, Oh JE, Hong J, et al. Optimized Biodegradable Polymeric ReservoirMediated Local and Sustained Co-Delivery of Dendritic Cells and Oncolytic Adenovirus Co-Expressing IL-12 and GM-CSF for Cancer Immunotherapy. J.Control.Release. 2017;259:115-127.

[28] Kim ID, Sawicki E, Lee HK, et al. Robust Neuroprotective Effects of Intranasally Delivered iNOS siRNA Encapsulated in Gelatin Nanoparticles in the Postischemic Brain. Nanomedicine. 2016;12(5):1219-1229.

[29] Sabet S, George MA, El-Shorbagy HM, et al. Gelatin Nanoparticles Enhance Delivery of Hepatitis C Virus Recombinant NS2 Gene. PLoS One. 2017;12(7):e0181723.

[30] Eke G, Mangir N, Hasirci N, et al. Development of a UV Crosslinked Biodegradable Hydrogel Containing Adipose Derived Stem Cells to Promote Vascularization for Skin Wounds and Tissue Engineering. Biomaterials. 2017;129:188-198.

[31] Oryan A, Alidadi S, Bigham-Sadegh A, et al. Effectiveness of Tissue Engineered Chitosan-Gelatin Composite Scaffold Loaded with Human Platelet Gel in Regeneration of Critical Sized Radial Bone Defect in Rat. J.Control.Release. 2017;254:65-74.

[32] Kang H, Shih YV, Hwang Y, et al. Mineralized Gelatin Methacrylate-Based Matrices Induce Osteogenic Differentiation of Human Induced Pluripotent Stem Cells. Acta Biomater. 2014;10(12):4961-4970.

[33] Feng Q, Wei K, Lin S, et al. Mechanically Resilient, Injectable, and Bioadhesive Supramolecular Gelatin Hydrogels Crosslinked by Weak HostGuest Interactions Assist Cell Infiltration and in Situ Tissue Regeneration. Biomaterials. 2016;101:217-228.

[34] Heo DN, Castro NJ, Lee SJ, et al. Enhanced Bone Tissue Regeneration using a 3D Printed Microstructure Incorporated with a Hybrid Nano Hydrogel. Nanoscale. 2017;9(16):5055-5062.

[35] He X, Feng B, Huang C, et al. Electrospun Gelatin/Polycaprolactone Nanofibrous Membranes Combined with a Coculture of Bone Marrow Stromal 
Cells and Chondrocytes for Cartilage Engineering. Int.J.Nanomedicine. 2015;10:2089-2099.

[36] Levato R, Webb WR, Otto IA, et al. The Bio in the Ink: Cartilage Regeneration with Bioprintable Hydrogels and Articular Cartilage-Derived Progenitor Cells. Acta Biomater. 2017;61:41-53.

[37] Brancato V, Comunanza V, Imparato G, et al. Bioengineered Tumoral Microtissues Recapitulate Desmoplastic Reaction of Pancreatic Cancer. Acta Biomater. 2017;49:152-166.

[38] Lee S, Serpooshan V, Tong X, et al. Contractile Force Generation by 3D hiPSC-Derived Cardiac Tissues is Enhanced by Rapid Establishment of Cellular Interconnection in Matrix with Muscle-Mimicking Stiffness. Biomaterials. 2017;131:111-120.

[39] Agrawal G, Aung A, Varghese S. Skeletal Muscle-on-a-Chip: An in Vitro Model to Evaluate Tissue Formation and Injury. Lab.Chip. 2017;17(20):34473461.

\section{Figure legends}

Figure 1: Some of the most promising approaches, being developed with gelatin as a primary biomaterial, including micro and nanoparticles for drug delivery, wound dressings, injectables, 3D scaffolds, bioinks and organs-on-achip technologies.

Figure 2: Hypertrophic scarring treatment with gelatin-based electrospun fibrous scaffolds. (A): Schematic illustration of the development of TGF- $\beta 1$ inhibitor-loaded PCL/ gelatin fibrous scaffolds. (B): Macroscopic evaluation of the wounds in different treatment groups (PGI: PCL/gelatin scaffold; PGT5: PCL/gelatin/TGF- $\beta 1$ inhibitor scaffold). Only PGT5 group showed a perfect restructuration of the wound without irregularities. (C): The healed wound area of each group at different observation time points. (D): Elevation index of scars in each group, harvested at 6 and 8 weeks. Two asterisks represent a significant difference of $p<0.01$. Three asterisks represent a significant difference of $p<0.001$. TGF- $\beta 1$ : Transforming growth factor $-\beta 1$; PCL: Polycaprolactone. Adapted with permission from Ref.[23] Copyright 2017 American Chemical Society. 
Figure 3: Bone tissue regeneration using a 3D printed microstructure incorporated with photo-curable gelatin hydrogel, functionalized with bioactive gold nanoparticles. (A): Schematic illustration of the therapeutic approach for personal bone tissue regeneration using 3D printing technology. (B): Gene expression levels of osteogenic differentiation markers of ADSCs cultured in the gelatin hydrogel $(\mathrm{Gel})$, gelatin hydrogel functionalized with gold nanoparticles (Gel-GNP), and gelatin hydrogel functionalized with RGD-conjugated gold nanoparticles (Gel-RGNP). ${ }^{*}$ indicates a significant difference of $p<0.05$. indicates a significant difference of $p<0.01$. (C): Optical images of calcium deposition staining from ADSCs culture in the three types of the hydrogel at 21 days. PLA: Polylactic acid; RGD: arginine-glycine-aspartate; ADSCs: human adipose-derived stem cells. Adapted from Ref [34] with permission from The Royal Society of Chemistry. 


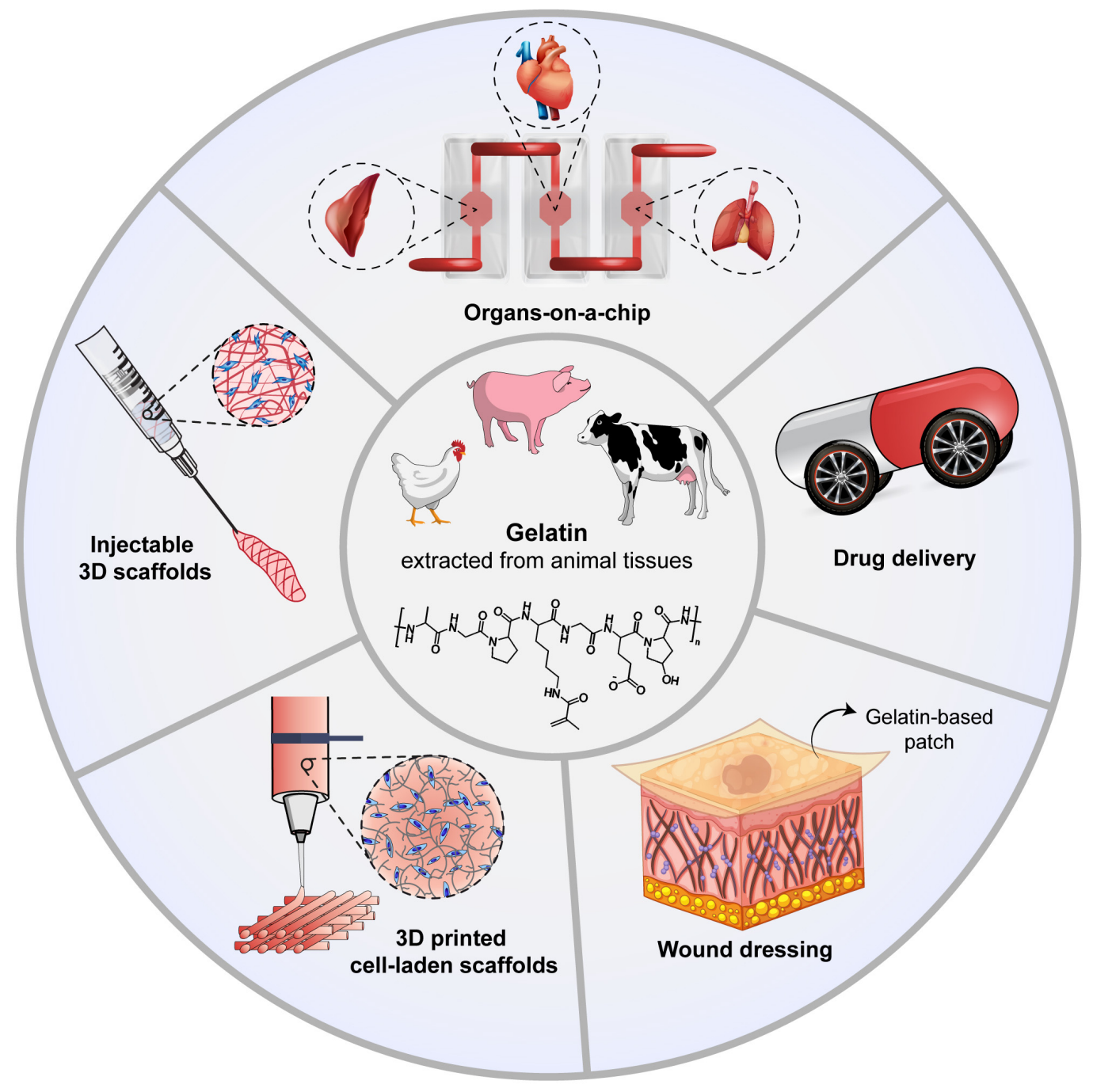

fig 1 
a
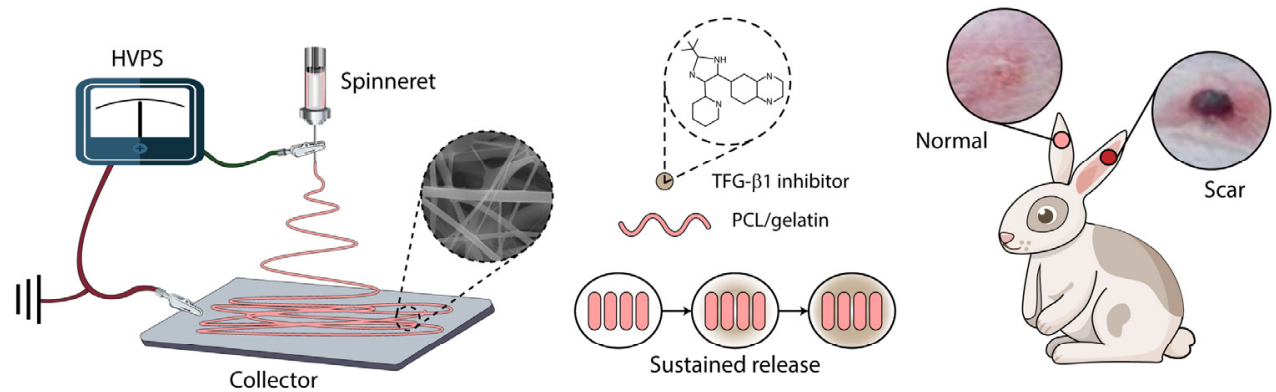

b

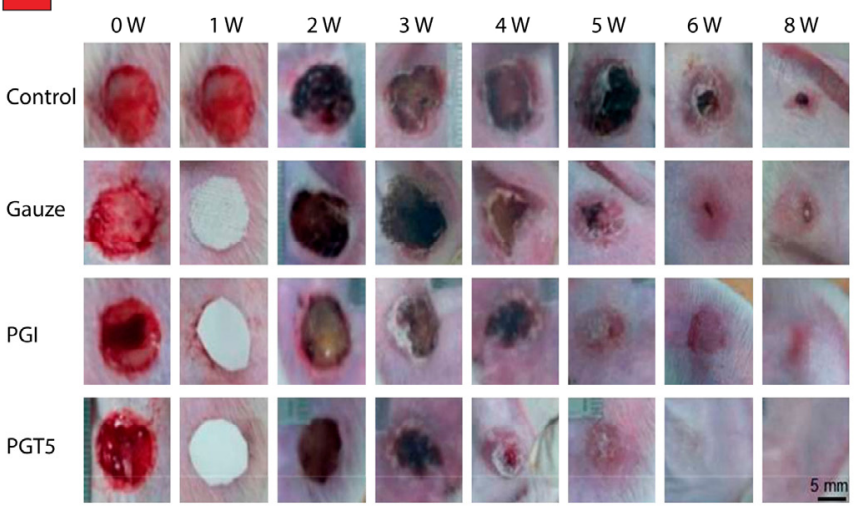

C

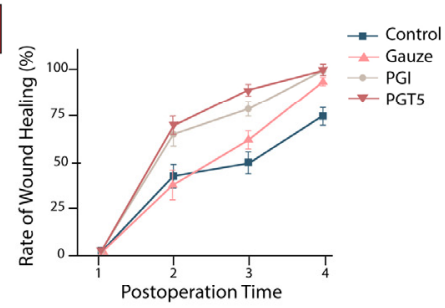

d

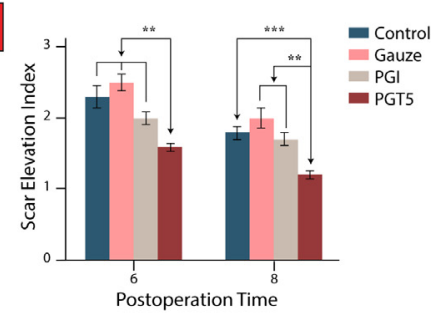

Fig 2 
a
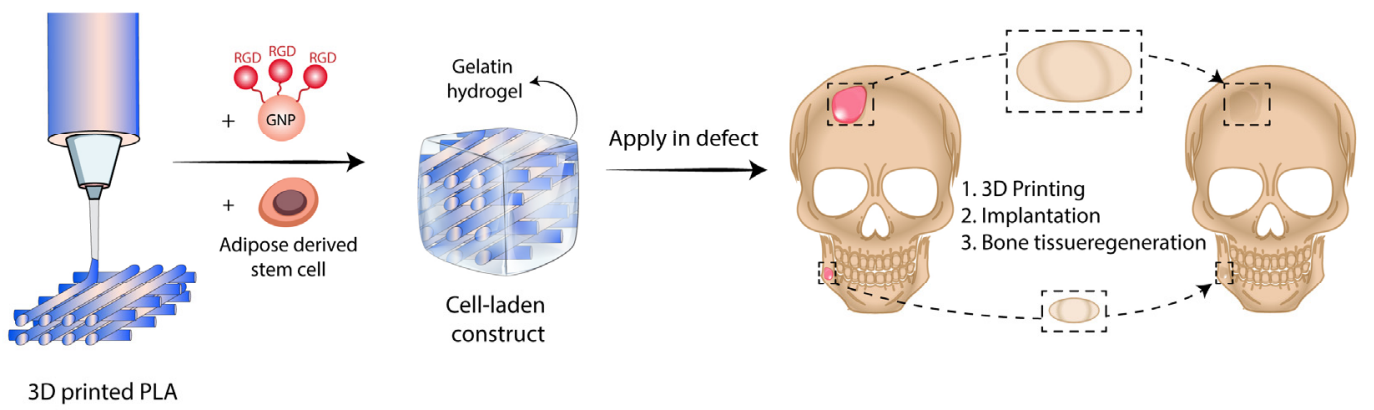

b
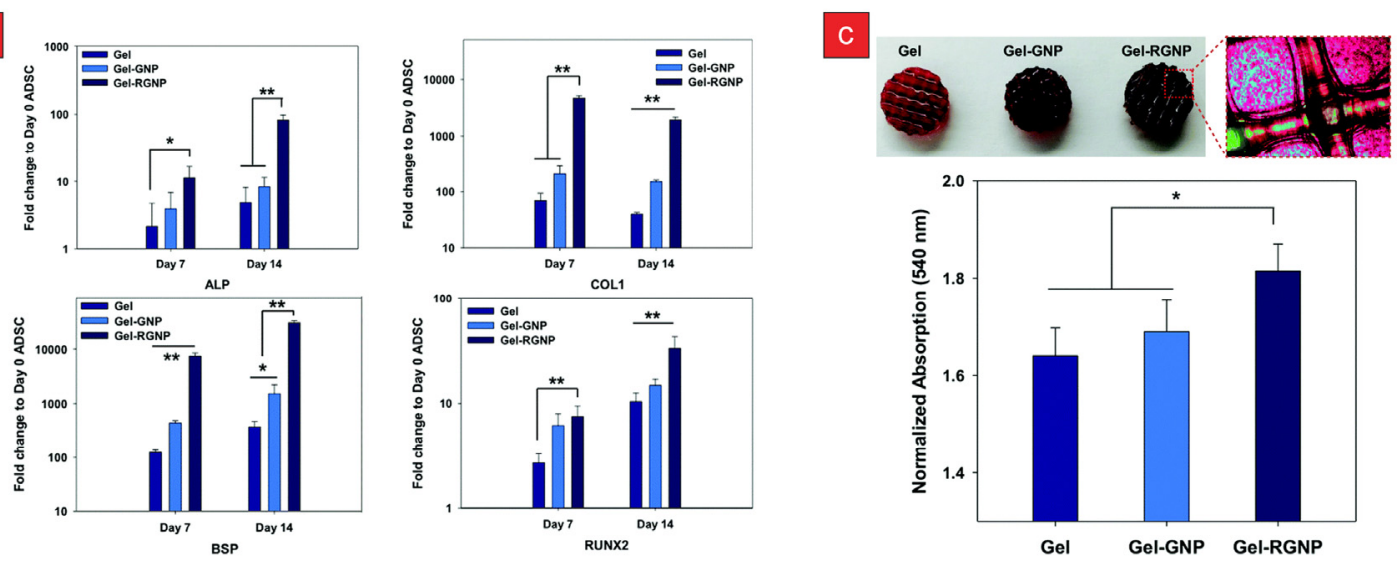

Fi g3 